\title{
A pilot study of dose-dense adjuvant paclitaxel without growth factor support for women with early breast carcinoma
}

\author{
Steven Sugarman - Carolyn Wasserheit - Elizabeth Hodgman · Maryellen Coglianese • \\ Anne D'Alassandro · Monica Fornier · Tiffany Troso-Sandoval · Gabriella D'Andrea • \\ Pamela Drullinsky $\cdot$ Diana Lake $\cdot$ Roshini George $\cdot$ Nancy Mills $\cdot$ Maryellen Moynahan • \\ Joyce Smith $\cdot$ Katherine Panageas $\cdot$ Larry Norton $\cdot$ Clifford Hudis
}

Published online: 22 July 2009

(C) Springer Science+Business Media, LLC. 2009

Erratum to: Breast Cancer Res Treat (2009) 115:609-612

DOI 10.1007/s10549-008-0152-9

Unfortunately the sixth author's name had been misspelled. The correct version is Monica Fornier and not Monica Fournier as published.

The online version of the original article can be found under doi:10.1007/s10549-008-0152-9.

S. Sugarman $(\bowtie) \cdot$ C. Wasserheit · E. Hodgman .

M. Coglianese - A. D'Alassandro · M. Fornier .

T. Troso-Sandoval · G. D’Andrea · P. Drullinsky · D. Lake ·

R. George · N. Mills · M. Moynahan - J. Smith - K. Panageas ·

L. Norton $\cdot$ C. Hudis

Memorial Sloan-Kettering Cancer Center, New York, NY, USA

e-mail: sugarmas@mskcc.org 